\title{
Designing the Magic Bullet? The Advancement of Immuno-PET into Clinical Use
}

\author{
Brian D. Wright and Suzanne E. Lapi \\ Department of Radiology, Washington University School of Medicine, St. Louis, Missouri
}

\begin{abstract}
The development of noninvasive imaging techniques using monoclonal antibodies (mAbs) is a quickly evolving field. Immuno-PET uses positron-emitting isotopes to track the localization of $m A b s$ with excellent image quality. Procedures for labeling mAbs with ${ }^{89} \mathrm{Zr}$ or ${ }^{124} \mathrm{I}$ using good manufacturing procedures have been established, and therefore these radiopharmaceuticals are being investigated for clinical use. This short review will focus on immuno-PET with full mAbs using long-lived positron-emitting isotopes $\left({ }^{89} \mathrm{Zr}\right.$ and $\left.{ }^{124} \mathrm{l}\right)$ over the past $5 \mathrm{y}$ and discuss their progress into clinical use.
\end{abstract}

Key Words: immuno-PET; ${ }^{124} \mathrm{I}$; ${ }^{89} \mathrm{Zr}$; PET; positron

J Nucl Med 2013; 54:1171-1174

DOI: 10.2967/jnumed.113.126086

The idea of the magic bullet was first suggested by Paul Ehrlich more than a century ago (1). He theorized that if a compound could target a disease selectively, then that compound could be combined with a therapeutic agent to treat the disease. This concept has inspired many drugs, both for treatment and for detection of a variety of diseases including cancer. Over the past 2 decades, targeted cancer therapy using monoclonal antibodies (mAbs) has shown that mAbs have many of the properties of Ehrlich's magic bullets. Currently, $22 \mathrm{mAbs}$ have been accepted by the U.S. Food and Drug Administration for therapy, with most being for the treatment of cancer (2). These molecules have high specificities for their targets and, when coupled with their high binding affinities, provide an attractive targeting tool to image and treat tumors.

Typically, mAbs achieve optimal tumor-to-nontumor ratios at 2-4 d after injection, which limits the choices of suitable radionuclides based on their physical half-lives (3). Common radiometals, such as ${ }^{99 \mathrm{~m}} \mathrm{Tc},{ }^{86} \mathrm{Y},{ }^{68} \mathrm{Ga}$, or ${ }^{64} \mathrm{Cu}$, have half-lives less than $15 \mathrm{~h}$, resulting in decay of the isotope before the attached radiolabeled mAbs achieve peak tumor-to-background ratios. However, ${ }^{64} \mathrm{Cu}$-labeled mAbs have found some success in clinical trials. Currently, phase I trials are investigating the relationship between ${ }^{64} \mathrm{Cu}$-labeled U3-1287 and human epidermal growth factor receptor 3 , as well as the dosimetry in humans. In a separate trial, the correlation between ${ }^{64} \mathrm{Cu}$-labeled trastuzumab uptake, tumor expression of human epidermal growth factor

Received Jun. 26, 2013; revision accepted Jul. 9, 2013.

For correspondence or reprints contact: Suzanne E. Lapi, Department of Radiology, Washington University School of Medicine, Campus Box 8225, St. Louis, MO 63110.

E-mail: lapis@mir.wustl.edu.

COPYRIGHT @ 2013 by the Society of Nuclear Medicine and Molecular Imaging, Inc. receptor 2 (HER2), and the inactivation of the phosphatidylinositol-3kinase/protein kinase B pathway are being investigated (4). The use of a longer-lived radionuclide enables the labeled $\mathrm{mAb}$ to achieve high tumor-to-background ratios before decay. ${ }^{124} \mathrm{I}$ and ${ }^{89} \mathrm{Zr}$, with half-lives of 4.18 and $3.27 \mathrm{~d}$, respectively, are good candidates for immuno-PET for this reason (5). The use of each isotope has its own advantages and disadvantages. Iodine has relatively simple and well-developed labeling chemistry; however, its decay involves high-energy positrons (687 and $974 \mathrm{keV}$ ), which decrease image resolution (6). The decay pathway of zirconium involves a lower positron energy emission; however, it also releases a high-energy, highly penetrating photon (909-keV $\gamma$-ray) $99 \%$ of the time. This photon means that more shielding and care in shipping and handling are required for ${ }^{89} \mathrm{Zr}$ than for many other PET isotopes. Additionally, the labeling chemistry is not as developed as that of iodine, usually using the conjugation of zirconium to a multidentate ligand such as deferoxamine (DFO) (Desferal; Macrocyclics). Furthermore, ${ }^{124} \mathrm{I}$ is commonly used with noninternalizing mAbs, which avoids dehalogenation from intracellular enzymes. ${ }^{89} \mathrm{Zr}$ is used with internalizing $\mathrm{mAbs}$ because it is a residualizing radionuclide and stays in the target cell after catabolism (7).

\section{LABELING CHEMISTRY}

Several exceptional reviews exist on the subject of immunoPET. An excellent review by Knowles and $\mathrm{Wu}$ gives readers a full introduction to the field (8). Koehler, Gagnon, and Wuest give an overview of the use of ${ }^{124} \mathrm{I}$, and a review by Deri and Lewis provides a comprehensive history of ${ }^{89} \mathrm{Zr}(9,10)$. In general, radioiodination of peptides and proteins is performed via the use of IODO-GEN (Pierce)-coated reaction vessels, eliminating the need to include the oxidizing agent in the reaction mixture and allowing for a simple filtration purification (11).

Radiolabeling of biomolecules with ${ }^{89} \mathrm{Zr}$ is most commonly performed through the modification of a native lysine side chain with activated esters of DFO. Until recently, most researchers applied Verel and van Dongen's method of conjugation using an activated 2,3,5,6-tetrafluorophenol ester of DFO. This method requires multiple steps, including protection of the DFO with iron, conjugation to the biomolecule, and then deprotection of the DFO (12). Recently, a much simpler method by Vosjan and van Dongen used p-isothiocyantobenzyl-DFO (Df-Bz-NCS) to conjugate DFO to the biomolecule in 1 step with good yields (13). This method lends itself to clinical translation because it requires fewer steps and the materials are commercially available.

\section{CLINICAL STUDIES: ${ }^{124} \mid$}

Several radioiodinated mAbs have recently been evaluated for clinical use. Carrasquillo et al. have evaluated a humanized A33 
(huA33) for PET imaging with ${ }^{124} \mathrm{I}$ (14). This mAb recognizes A33 antigen, which is known to be expressed in greater than $95 \%$ of human colon adenocarcinomas. In this study, 25 patients with primary or metastatic colorectal cancer (CRC) were administered 44.4$396 \mathrm{MBq}$ (median, $343 \mathrm{MBq}$ ) of ${ }^{124} \mathrm{I}$-huA33 with a total of $10 \mathrm{mg}$ of huA33. The authors observed no adverse side effects during the treatment that could be attributed to the huA33. Treatment could be administered via intravenous administration or hepatic arterial infusion (HAI), with HAI giving no detectable advantage over intravenous injection. Eleven patients had 12 primary tumors, 10 of which were observed via immuno-PET. Ten patients had liver metastases, all of which were identified by ${ }^{124} \mathrm{I}$-huA33 (Fig. 1). Four of 7 patients with nodal metastases displayed uptake of the ${ }^{124} \mathrm{I}-\mathrm{huA} 33$, and 2 of 5 patients with lung lesions were visualized by immuno-PET. Furthermore, the tracer uptake in the tumors displayed an extended retention time, and in some cases this retention was so long the authors were unable to determine the time required for clearance. Even with this issue, the study was still in agreement with the half-life that had been reported by others, and displayed an average clearance of $3.10 \pm 5.6$ $\mathrm{mL} / \mathrm{h}$ for intravenous injection, $36.8 \pm 8.4 \mathrm{~mL} / \mathrm{h}$ for intravenous $\mathrm{IgG}$, and $35.2 \pm 8.0 \mathrm{~mL} / \mathrm{h}$ for HAI (14). The authors also note that even with the superior localization of the ${ }^{124} \mathrm{I}-\mathrm{A} 33$ in the tumor in comparison to the colon tissue (4:1 tumor-to-colon ratio), there was some difficulty in identifying the primary colon tumors and lymph node lesions because of high background activity. They suggest that this problem could be remedied by delaying imaging further to allow for clearance of the tracer from the bowel.

Although intact mAbs do not cross the blood-brain barrier, imaging and treatment of metastatic tumors is possible because of a compromised blood-brain barrier. In a recent investigation, Poli et al. evaluated ${ }^{124}$ I-labeled L19SIP (radretumab) for use in determining provisional doses of ${ }^{131}$ I-labeled radretumab in 6 patients with brain metastasis ( 3 from non-small cell lung cancer and 3 from breast carcinoma). Radretumab, a fully humanized $\mathrm{mAb}$, has been thoroughly investigated previously as a ${ }^{131} \mathrm{I}$-labeled targeting agent (15). This mAb targets an epitope contained in the extra-domain B of fibronectin, a domain rarely found in normal adult tissue but often found in the extracellular matrix surrounding newly formed blood vessels in tumors (16). In this study, each of the 6 patients was administered an average of $167 \mathrm{MBq}$ (roughly $3.3 \mathrm{mg}$ of protein), and the bone red marrow, tumor lesion, and healthy organ doses were monitored. The bone red marrow dose was found

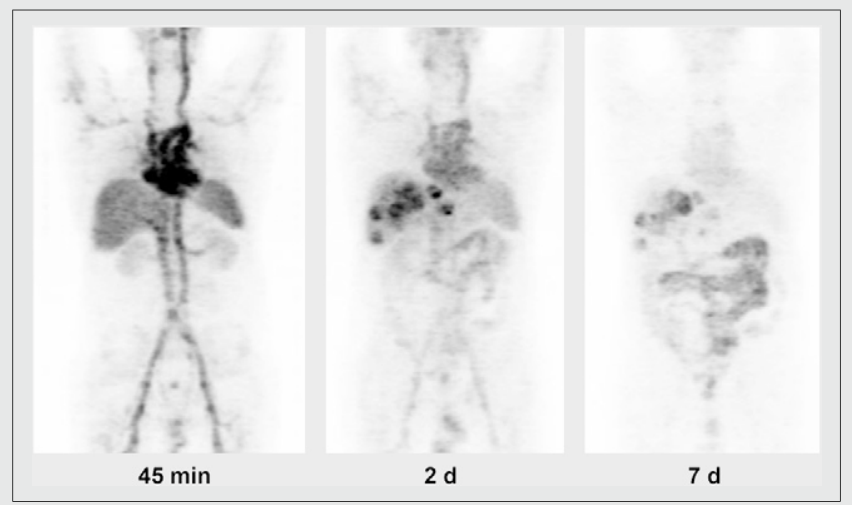

FIGURE 1. Example of ${ }^{124}$ |-huA33 imaging in patient with $\mathrm{CRC}$ metastasized to liver. Images show blood-pool activity at $45 \mathrm{~min}$, excellent localization in liver lesions at $2 \mathrm{~d}$, and persistent uptake in liver lesions at $7 \mathrm{~d}$. (Reprinted with permission of (14).) to be approximately $0.2 \mathrm{~Gy} / \mathrm{GBq}$ and always less than $2 \mathrm{~Gy}$, suggesting that up to $7.4 \mathrm{GBq}$ could be injected without compromising the blood-building system long-term. These results correlated favorably with clinical findings for ${ }^{131} \mathrm{I}$-radretumab ( $\leq 9.3 \mathrm{GBq}$ allowed) (15). The absorbed lesion dose was observed to be an average of 2.4 Gy (range, 0.70-8.1 Gy) for brain metastases and 7.3 Gy (range, 1.1-35.8 Gy) for extracranial lesions. The absorbed dose in the healthy organs was constant among all 6 patients. Only the thyroid displayed variable uptake among patients; however, this variability was explained by the fact that 3 of the patients did not strictly follow the thyroid-blocking therapy that was prescribed and is attributed to dehalogenation.

In a study published early this year, Divgi et al. reported the results of a multicenter phase III clinical study evaluating the effectiveness of ${ }^{124}$ I-labeled cG250 (girentuximab) in detecting clear cell renal cell carcinoma (ccRCC) (17). This chimeric antibody binds carbonic anhydrase IX (CAIX), which is expressed in more than $95 \%$ of ccRCC. The ${ }^{124}$ I-girentuximab was administered to 204 patients at 14 centers in the United States and was monitored by PET/CT and contrast-enhanced CT (CECT). Of the 204 patients, 203 underwent CECT and 202 had surgery to confirm or remove the tumors. No allergic reactions to the drug were observed in the patients during the study; however, adverse effects to the treatment were reported in 30 of the patients $(13.3 \%)$. The most commonly reported event was headache (10 patients, $4.4 \%)$. The authors reported that 1 patient had a grade 3 agent-related event (transient liver enzyme increase); however, the patient had also received ciprofloxacin before the event. The average sensitivity and specificity for detection of ccRCC were $86.2 \%$ and $85.9 \%$, respectively, and all lesions below $1 \mathrm{~cm}$ were visualized. In comparison, the average sensitivity and specificity for CECT were $75.5 \%$ and $46.8 \%$, respectively. Additionally, the accuracy of the PET/CT estimates was consistently greater than that of the CECT (range, 85.6\%-86.7\%, compared with $66.2 \%-69.2 \%$ ). This study confirmed the efficacy of ${ }^{124} \mathrm{I}-$ girentuximab as a noninvasive method of identifying ccRCC. Although the requirement for participation in the study was that patients be able to undergo surgery, the imaging strategy has the potential to reduce unnecessary surgeries in patients with benign renal masses.

\section{PRECLINICAL PROGRESS: ${ }^{89}$ ZR}

The success of the earlier phase I study of Divgi et al. inspired Stillebroer et al. to investigate the comparison of ${ }^{124}$ I-labeled girentuximab and ${ }^{89} \mathrm{Zr}$-labeled girentuximab (18). In this study, nude mice with subcutaneous ccRCC xenografts were treated with either ${ }^{89} \mathrm{Zr}$-Df-girentuximab or ${ }^{124} \mathrm{I}$-girentuximab, and the biodistribution was observed $7 \mathrm{~d}$ after injection. Uptake of ${ }^{89} \mathrm{Zr}$-Df-girentuximab was significantly higher than that of ${ }^{124}$ I-girentuximab in CAIX-expressing tumors $(114.7 \pm 25.2$ percentage injected dose [\%ID] vs. $38.2 \pm 18.3 \%$ ID) but was similar in the CAIX-negative tumors $(48.7 \pm 15.2 \%$ ID vs. $32.0 \pm$ $22.9 \%$ ID). Images of the ${ }^{89} \mathrm{Zr}$-Df-girentuximab tumors had higher contrast and less noise than those of ${ }^{124}$ I-girentuximab. Comparison of the biodistribution in both models displayed a higher amount of ${ }^{89} \mathrm{Zr}$-Df-girentuximab in the liver and spleen, whereas the ${ }^{124}$ I-girentuximab scans showed higher activity in the thyroid. Additionally, the tumor uptake was assessed by comparing the ${ }^{89} \mathrm{Zr}$-Df-girentuximab with an irrelevant control mAb $\left({ }^{89} \mathrm{Zr}\right.$ Df-MOPC21). Uptake of ${ }^{89} \mathrm{Zr}$-Df-girentuximab in the CAIX-positive tumors was greater than that of the irrelevant $\mathrm{mAb}(36.5 \pm 6.2 \% \mathrm{ID}$ vs. $6.8 \pm 2.3 \% \mathrm{ID})$, showing that the uptake was due to the 
affinity of the mAb for CAIX. The high specificity and greater contrast demonstrated here, combined with the success of the recent phase III trial by Divgi et al., show promise for ${ }^{89} \mathrm{Zr}$ Df-girentuximab.

Development of a mAb that targets a receptor commonly overexpressed by multiple tumor types would allow for a more general treatment for patients. The epidermal growth factor receptor (EGFR), a member of the ErbB tyrosine kinase receptor family, is known to be overexpressed in most tumors (19). Panitumumab is a fully humanized antibody that is approved by the Food and Drug Administration for treatment of metastatic CRC; however, it has been demonstrated that panitumumab can be effective against other malignancies as well $(20,21)$. EGFR expression can vary between primary and metastatic tumors, and thus a noninvasive method for determining EGFR expression in patients would allow for a more accurate dosing regimen. Attempting to solve this issue, both Nayak et al. (20) and Chang et al. (21) have explored the use of panitumumab labeled with ${ }^{89} \mathrm{Zr}$ with promising results. Nayak et al. investigated the efficacy of imaging with ${ }^{89} \mathrm{Zr}$ panibumumab in 2 CRC xenografts (LS-174T and A375 cells). Imaging results showed high uptake in the highly EGFR-expressing LS-174T and low uptake in the EGFR-negative A375 cells (20). In the study by Chang et al., ${ }^{89} \mathrm{Zr}$-panitumumab imaging was examined in 4 different xenografts (A431, HTC116, MDA-MB435, and T47D cells) of varying levels of EGFR expression. The imaging results showed high uptake in the highly EGFR-expressing A431 tumors and moderate intensity in the moderately expressing HCT116 tumors. The intensity for the low and nonexpressing tumors was minimal. Cold panitumumab was shown to block uptake in both HCT116 tumors (21) and LS-174T tumors (20), demonstrating a significant decrease in intensity after $5 \mathrm{~d}$ and illustrating the specificity of the tracer. Use of this radiotracer would enable EGFR expression to be quantified in patients to determine the effectiveness of this type of treatment.

\section{CLINICAL STUDIES: ${ }^{89}$ ZR}

Several researchers have investigated ${ }^{89} \mathrm{Zr}$-labeled $\mathrm{mAbs}$ in a clinical setting. Böjesson et al. investigated the safety of a chimeric $\mathrm{mAb}$ known as U36 labeled with ${ }^{89} \mathrm{Zr}$ to target the v6 region of CD44 of head and neck squamous cell carcinoma (22). This study was a follow-up to their earlier work, in which they demonstrated the diagnostic capabilities of this radiolabeled antibody. Twenty patients underwent imaging with ${ }^{89} \mathrm{Zr}$-U36 before surgery $(10 \mathrm{mg}, 74.9$ $\mathrm{MBq}$ ). From the study, Böjesson et al. determined that the ${ }^{89} \mathrm{Zr}$ U36 was safe for all subjects. No adverse reactions were observed during the study. The mean dose for patients, about $40 \mathrm{mSv}$, will limit the number of times a patient can repeat the treatment. The authors do suggest that the use of newer PET/CT scanners will give better images, halve the mean dose, and reduce the number of treatments required.

Trastuzumab (Herceptin; Genentech) targets the HER2, a wellresearched receptor known for its involvement in cell proliferation, metastasis, and angiogenesis (23). Dijkers et al. conducted the firstin-human investigation of ${ }^{89} \mathrm{Zr}$-trastuzumab in 2010 , with the goal of determining the optimal dosage and time of administration. Fourteen patients were administered $38.4 \pm 1.6 \mathrm{MBq}$ of ${ }^{89} \mathrm{Zr}$-trastuzumab, and no reactions or adverse events were observed during the study. Patients with HER2-positive metastatic breast cancer were split into 3 groups and administered either $10 \mathrm{mg}$ of ${ }^{89} \mathrm{Zr}$-trastuzumab, $50 \mathrm{mg}$ of ${ }^{89} \mathrm{Zr}$-trastuzumab, or $10 \mathrm{mg}$ of ${ }^{89} \mathrm{Zr}$-trastuzumab plus trastuzumab therapy and then were imaged after $5 \mathrm{~d}$. Lesions were visualized in only one of the patients receiving $10 \mathrm{mg}$ of the imaging agent only. A high uptake in the liver and a prominent intestinal excretion were observed, which corresponded with previous pharmacokinetic studies (23). Trastuzumab-naïve patients treated with $50 \mathrm{mg}$ of ${ }^{89} \mathrm{Zr}$ trastuzumab showed decreased uptake in the liver in comparison to the 10-mg dose and an increased presence in the blood pool, suggesting retarded blood clearance. Patients undergoing trastuzumab treatment in addition to the $10 \mathrm{mg}$ of ${ }^{89} \mathrm{Zr}$-trastuzumab also displayed a decreased blood clearance. Most of the metastatic lesions were visualized and confirmed via CT and MR imaging in both 50-mg and 10-mg-plus-therapy groups; however, it was noted that in 6 of 12 patients, not all known lesions were detected. The authors suggest that this is due to varying expression of HER2 between lesions or from suboptimal imaging conditions. Unexpectedly, Dijkers et al. were able to visualize brain lesions with an 18fold-higher uptake in the tumors than in normal tissues and were able to discover previously undetected lesions that were later confirmed by MR imaging (Fig. 2). This suggests that the trastuzumabbased therapies can be used to treat patients with HER2-positive brain metastases. Several clinical trials are currently under way investigating ${ }^{89} \mathrm{Zr}$-labeled trastuzumab as a diagnostic tool for HER2-positive metastases. Additionally, several more clinical trials are looking at other ${ }^{89} \mathrm{Zr}$-labeled mAbs, such as cetuximab and bevacizumab, as PET imaging agents (24).

\section{CONCLUSION}

The field of immuno-PET is rapidly progressing toward clinical use. ${ }^{124}$ I has been shown to be a long-lasting and effective radionuclide for imaging various types of lesions, both primary and metastatic, and for determining the effectiveness of ${ }^{131}$ I treatments. Phase III clinical trials using ${ }^{124}$ I-girentuximab have been shown to be an effective method for detecting ccRCC in more than 200 patients. ${ }^{89} \mathrm{Zr}$-labeled mAbs, although still requiring more research, have shown promising results in targeting a wide variety of receptors and tumor types. In an effort to hasten the localization of the radiometals, some researchers have also looked to engineered $\mathrm{mAb}$ fragments, diabodies, minibodies, and other $\mathrm{mAb}$ fragments Future work in the field will likely improve current procedures and make use of these other engineered targeting moieties to improve imaging capabilities.

\section{DISCLOSURE}

This work is supported in part by DOE grant DESC0008432. No other potential conflict of interest relevant to this article was reported.

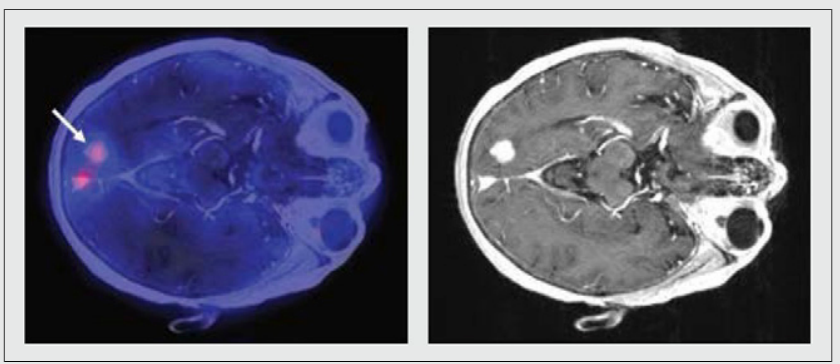

FIGURE 2. Example of HER2-positive brain lesion undetected by conventional scans, revealed with ${ }^{89} \mathrm{Zr}$-trastuzumab imaging and subsequently confirmed by MR imaging. Arrow indicates lesion. (Reprinted with permission of (23).) 


\section{ACKNOWLEDGMENTS}

We thank the Lapi Research Group for useful discussions.

\section{REFERENCES}

1. Ehrlich C. Experimental Researches on Specific Therapy: On Immunity with Special Reference to the Relationship Between Distribution and Action of Antigens. Royal Institute of Public Health. 107th ed. London, U.K.: Lewis; 1908.

2. Scolnik PA. mAbs: a business perspective. MAbs. 2009;1:179-184.

3. van Dongen GAMS, Vosjan MJWD. Immuno-positron emission tomography: shedding light on clinical antibody therapy. Cancer Biother Radiopharm. 2010;25:375-385.

4. Mortimer JE. National Cancer Institute at the National Institutes of Health. Positron emission tomography in women with advanced HER2-positive breast cancer. http://www.cancer.gov/clinicaltrials/search/view?cdrid=669649\&version $=$ Health Professional\&protocolsearchid=11815781. Accessed July 9, 2013.

5. Wadas TJ, Wong EH, Weisman GR, Anderson CJ. Coordinating radiometals of copper, gallium, indium, yttrium and zirconium for PET and SPECT imaging of disease. Chem Rev. 2010;110:2858-2902.

6. Ruggiero A, Holland JP, Lewis JS, Grimm J. Cerenkov luminescence imaging of medical isotopes. J Nucl Med. 2010;51:1123-1130.

7. Verel I, Visser GWM, Boerman OC, et al. Long-lived positron emitters zirconium89 and iodine-124 for scouting of therapeutic radioimmunoconjugates with PET. Cancer Biother Radiopharm. 2003;18:655-661.

8. Knowles SM, Wu AM. Advances in immuno-positron emission tomography: antibodies for molecular imaging in oncology. J Clin Oncol. 2012;30:38843892 .

9. Koehler L, Gagnon K, McQuarrie S, Wuest F. Iodine-124: a promising positron emitter for organic PET chemistry. Molecules. 2010;15:2686-2718.

10. Deri MA, Zeglis BM, Francesconi LC, Lewis JS. PET imaging with ${ }^{89} \mathrm{Zr}$ : from radiochemistry to the clinic. Nucl Med Biol. 2013;40:3-14.

11. Fraker PJ, Speck JC. Protein and cell membrane iodinations with a sparingly soluble chloroamide, 1,3,4,6-tetrachloro-3a, 6a-diphenylglycoluril. Biochem Biophys Res Commun. 1978;80:849-857.
12. Verel I, Visser GWM, Boellaard R, Walsum MS, Snow GB, van Dongen GAMS. ${ }^{89} \mathrm{Zr}$ immuno-PET: comprehensive procedures for the production of ${ }^{89} \mathrm{Zr}$-labeled monoclonal antibodies. $J$ Nucl Med. 2003;44:1271-1281.

13. Vosjan MJWD, Perk LR, Visser GWM, et al. Conjugation and radiolabeling of monoclonal antibodies with zirconium-89 for PET imaging using the bifunctional chelate p-isothiocyanatobenzyl-desferrioxamine. Nat Protoc. 2010;5:739-743.

14. Carrasquillo JA, Pandit-Taskar N, O'Donoghue JA, et al. ${ }^{124} \mathrm{I}-\mathrm{huA} 33$ antibody PET of colorectal cancer. J Nucl Med. 2011;52:1173-1180.

15. Poli GL, Bianchi C, Virotta G, et al. Radretumab radioimmunotherapy in patients with brain metastasis: a ${ }^{124}$ I-L19SIP dosimetric PET study. Cancer Immunol Res. 2013:OF1-OF10.

16. Schliemann C, Neri D. Antibody-based vascular tumor targeting. Recent Results Cancer Res. 2010;180:201-216.

17. Divgi CR, Uzzo RG, Gatsonis C, et al. Positron emission tomography/computed tomography identification of clear cell renal cell carcinoma: results from the REDECT Trial. J Clin Oncol. 2013;31:187-194.

18. Stillebroer AB, Franssen GM, Mulders PFA, et al. ImmunoPET imaging of renal cell carcinoma with ${ }^{124} \mathrm{I}$ - and ${ }^{89} \mathrm{Zr}$-labeled anti-CAIX monoclonal antibody cG250 in mice. Cancer Biother Radiopharm. May 22, 2013 [Epub ahead of print].

19. Lurje G, Lenz HJ. EGFR signaling and drug discovery. Oncology. 2009;77: $400-410$.

20. Nayak TK, Garmestani K, Mielenic DE, Brechbiel MW. PET and MR imaging of metastatic peritoneal and pulmonary colorectal cancer in mice with human epidermal growth factor receptor 1-targeted ${ }^{89} \mathrm{Zr}$-Labeled panitumumab. J Nucl Med. 2012;53:113-120.

21. Chang AJ, DeSilva RA, Lapi SE. Development and characterization of ${ }^{89} \mathrm{Zr}-$ labeled panitumumab for immuno-positron emission tomographic imaging of the epidermal growth factor receptor. Mol Imaging. 2013;12:17-27.

22. Börjesson PKE, Jauw YWS, de Bree R, et al. Radiation dosimetry of ${ }^{89} \mathrm{Zr}$ labeled chimeric monoclonal antibody U36 as used for immuno-PET in head and neck cancer patients. $J$ Nucl Med. 2009;50:1828-1836.

23. Dijkers EC, Oude Munnink TH, Kosterink JG, et al. Biodistribution of ${ }^{89} \mathrm{Zr}$ trastuzumab and PET imaging of HER2-positive lesions in patients with metastatic breast cancer. Clin Pharmacol Ther. 2010;87:586-592.

24. ${ }^{89} \mathrm{Zr}$. http://www.cancer.gov/clinicaltrials/search/results?protocolsearchid $=11815785$. Accessed July 15, 2013 